\title{
Setting the Parameters Right for Two-hop IEEE 802.11e Ad Hoc Networks
}

\author{
Anne Remke ${ }^{1}$, Boudewijn R. Haverkort ${ }^{1,2}$, Geert Heijenk ${ }^{1}$ and Jesper Bax ${ }^{1}$ \\ 1 University of Twente \\ Design and Analysis of Communication Systems \\ Enschede, The Netherlands \\ http://dacs.cs.utwente.nl/ \\ 2 Embedded Systems Institute \\ Eindhoven, The Netherlands \\ http://www.esi.nl
}

\begin{abstract}
Two-hop ad-hoc networks, in which some nodes forward traffic for multiple sources, with which they also compete for channel access suffer from large queues building up in bottleneck nodes. This problem can often be alleviated by using IEEE 802.11e to give preferential treatment to bottleneck nodes. Previous results have shown that differentiation parameters can be used to allocate capacity in a more efficient way in the two-hop scenario. However, the overall throughput of the bottleneck may differ considerably, depending on the differentiation method used. By applying a very fast and accurate analysis method, based on steady-state analysis of an QBD-type infinite Markov chain, we find the maximum throughput that is possible per differentiation parameter. All possible parameter settings are explored with respect to the maximum throughput conditioned on a maximum buffer occupancy. This design space exploration cannot be done with network simulators like NS2 or Opnet, as each simulation run simply takes to long.

The results, which have been validated by detailed simulations, show that by differentiating TXOP it is possible to achieve a throughput that is about $50 \%$ larger than when differentiating AIFS and $\mathrm{CW}_{\min }$.
\end{abstract}

\section{Introduction}

The availability of cheap yet powerful wireless access technology, most notably IEEE 802.11 ("wireless LAN"), has given an impulse to the development of wireless ad hoc networks. In such networks, the stations (nodes) that are in reach of each other, help each other in obtaining and maintaining connectivity. At the same time they are also competitors, as they all contend for the same resource, i.e., the shared ether as transmission medium. The medium access control of IEEE 802.11 (based on CSMA/CA) is commonly referred to as the distributed

\footnotetext{
* The work presented in this paper has been performed in the context of the $\mathrm{MC}=\mathrm{MC}$ project (612.000.311), financed by the Netherlands Organization for Scientific Research (NWO).
} 
coordination function (DCF) $[6,12]$. Research has shown that, effectively, the DCF tends to equally share the capacity among contending stations $[2,7]$. Although this appears to be a nice fairness property, this fairness does lead to undesirable situations in case one of the nodes that functions as bridge toward either another group of nodes, or via an access point to the wired internet, as illustrated in Figure 1. In such cases, it appears fair to allocate more bandwidth to the bridging node.

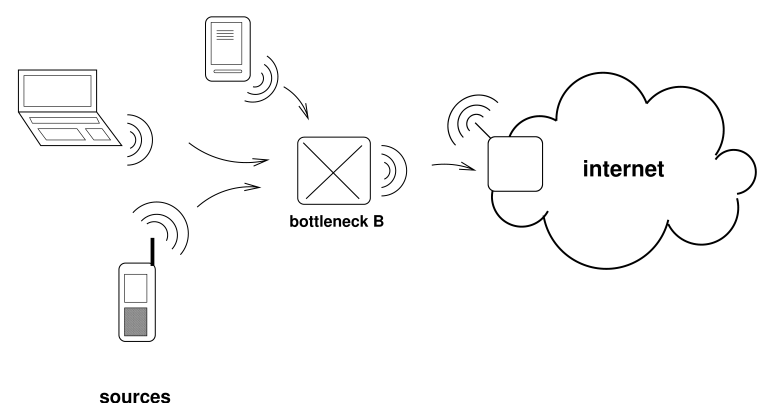

Fig. 1. Bottleneck in a two-hop ad hoc network

Recently, a quality-of-service (QoS)-extension of the IEEE 802.11 standard, the so-called EDCA ("e") version has been released [1]. Roughly speaking, this extension provides mechanisms to provide preferential treatment of certain traffic classes (or nodes) over others. Four different parameters can be used to reallocate the amount of radio capacity given to each station, corresponding to a large number of different parameter settings.

The current paper analyzes how we can optimize IEEE 802.11e parameter settings such that maximum throughput is obtained for a given buffer size in this 2-hop ad hoc network scenario?

Even though single parameter settings can be simulated with network simulators like NS2 or Opnet, it is practically impossible to find the optimal parameter setting for a given scenarios using costly and slow simulations. In contrast to the simulations a very fast analytical approach has been proposed in [10], where we presented a new model for analyzing IEEE 802.11e access mechanism in a two-hop ad hoc network. Our high-level model is flow-based, and uses results from packet-based models (such as those proposed by Bianchi and Engelstad et al. $[2,4])$, and allows for the numerical evaluation of the buffer occupancy at the bottleneck node, the system throughput, as well as provides information on the mean number of active sources. This analytical approach has been verified by extensive simulations.

However, the key result of the current paper is that we use these models in a variety of scenarios, and show how they can be used to optimize system parameters. By exploring the parameter choices for all stations and the chosen 
threshold for the buffer occupancy, we obtain the maximum load parameter $\lambda$ for which the buffer occupancy still remains below the threshold and then compute the throughput that corresponds to this load. Note that we only differentiate one parameter at a time.

This cannot be achieved with detailed simulation models, simply because they are too time-consuming to be executed so often. We do, however, show that also for the optimized parameter scenarios, the results obtained with the analytical models, do coincide very well with detailed simulation studies.

The only paper we are aware of explicitly addressing an analytical evaluation of the two-hop case is [13]. They obtain explicit (closed-form) equations for the expected overall delay and the expected delay at the bottleneck by translating the model at hand into a generalized processor sharing model, as studied by Cohen [3]. Although the analysis is approximate, good results are obtained, as confirmed by simulations. However, this evaluation approach is limited in that it only allows for an equal sharing of transmission capacity between all active stations (including the bottleneck). They do not address the differentiation parameters introduced in the protocol IEEE 802.11e.

In this paper, the system of interest and the modeling approach is described in Section 2. We compare the maximum throughput that can be obtained with the different QoS parameters with the maximum obtainable throughput in the basic setting in Section 3. Conclusions and future work are discussed in Section 4.

\section{System of interest and modeling approach}

In Section 2.1 we describe the scenario of interest, in Section 2.2 the quality of service parameters are described and in Section 2.3 the analytical modeling approach is discussed.

\subsection{Bottleneck scenario}

The scenario under study, as illustrated in Figure 1, has a varying number $N$ of active nodes, the so-called sources, which are all within reach of each other. Additionally there is the bottleneck node $B$, that is the only node that can reach via an access point the wired internet. Hence, all traffic originating from the sources and the traffic passing through the bridge has to share the same radio transmission capacity. It has been shown that the DCF access mechanism effectively shares the radio capacity equally over all competing nodes [2,7]. Clearly, this situation benefits the sources as a group, as they can use a relatively large share of radio capacity to send their packets, whereas the bottleneck only gets the same share as every other individual node. Because it has to support the traffic of all other nodes, fairness leads to a very high buffer occupancy in $B$, eventually also buffer overflow, and in any case, long delays. 


\section{$2.2 \quad$ IEEE $802.11 \mathrm{e}$}

The Enhanced Distributed Channel Access Function (EDCA) of IEEE 802.11e allows multiple contention instances to be simultaneously active in a single station, each supporting a certain access category (AC). Furthermore, the standard introduces four differentiation parameters (EDCA parameters), as discussed below, which can be set individually for each access category of each individual station to enable QoS provisioning [11].

We facilitate adaptive capacity sharing between stations by letting each station have a single access category, and using the EDCA parameters for differentiating between the source stations and the bottleneck station. In principle the EDCA parameters are meant for service differentiation, while we apply it here for node differentiation. Another relevant scenario for such node level differentiation is the case of uplink versus downlink transfer in an infrastructure-based WLAN, where the access point should get a bigger share of the resources to achieve fairness between both directions $[9,5]$. In the remainder of this paper we will analyze the following four scenarios:

1. With standard IEEE 802.11 , the medium needs to be idle for at least one distributed inter-frame spacing (DIFS) period before stations can start to contend for medium access. A station then needs to wait a random number of slots, drawn from the so-called contention window $(\{0, \mathrm{CW}\})$, before starting to transmit if the medium is still idle. After winning contention a station is allowed to send exactly one packet. The range of the window grows with every collision (until the maximum is reached) and is reset to its minimum after a successful transmission.

In the IEEE 802.11e QoS extension, two contention-based methods are proposed to change the above procedure:

2. The initial value of the contention window $\left(\mathrm{CW}_{\min }-1\right)$ and/or the maximum value of the contention window $\left(\mathrm{CW}_{\max }-1\right)$ are set smaller for a given station, thus, this station draws its backoff from a smaller contention window, hence, has a higher probability to win contention.

3. With so-called arbitration inter-frame spacing (AIFS) it is possible to assign different inter-frame spacings for different service classes (or nodes) instead of the fixed DIFS. Thus, high-priority nodes can be assigned shorter AIFS, so that they can start counting off their backoff earlier, hence, have an advantage when contending for medium access.

A way to adapt the capacity sharing that does not alter the actual contention mechanism is the following:

4. The transmission opportunity limit $\left(\mathrm{TXOP}_{\text {limit }}\right)$ provides a time period during which a station may send packets after having won a contention. Thus, a station with a sufficiently high $\mathrm{TXOP}_{\text {limit }}$ is able to send several packets and will thus be able to grab a larger share of the channel capacity than a station with a smaller $\mathrm{TXOP}_{\text {limit }}$. 
The above four parameters $\left(\mathrm{CW}_{\min }\right.$ and $\mathrm{CW}_{\max }$, AIFS and $\left.\mathrm{TXOP}_{\text {limit }}\right)$ in the IEEE 802.11e standard can be used to reallocate the amount of radio capacity given to the sources and to the bottleneck.

\subsection{An abstract analytical model}

In this section we briefly recall the analytical model, for a more detailed description please refer to [10].

We model the bottleneck $B$, cf. Figure 1, using an infinite-state stochastic Petri net (iSPN), as given in Figure 2. The left part of this figure contains an unbounded place (double circle) buffer that models the (buffer of the) bottleneck of the system. Transition input models the total arrival stream of packets from all active sources, whereas transition output models the transmission of packets leaving the bottleneck $B$. We limit the maximum number of active sources to some finite number $K$ and do not distinguish between individual active sources, modeled by the right part of the iSPN in Figure 2. An inactive source becomes

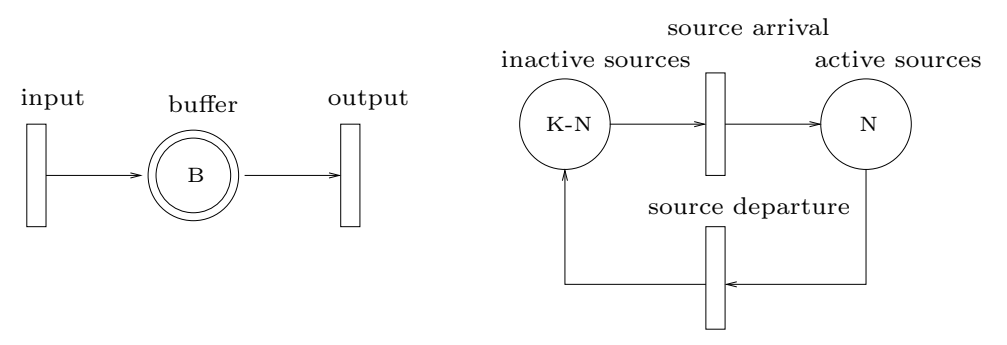

Fig. 2. High-level model as iSPN

active after a negative exponentially distributed amount of time (with mean $1 / \lambda$ ) and immediately instantiates a flow, which has a geometrically distributed length, measured in packets. The average size of a data packet is assumed to be $E[P]=1500$ bytes, with exponentially distributed length. The duration of a flow does not only depend on its size but also on the radio capacity a source can use to transmit the flow. Note that the duration of a flow implicitly gives the source departure rate, as well. Hence, the behavior of the sources depends on the system behavior. This traffic model is realistic for interactive applications, such as web browsing. Following the parametric assumptions made in [13], the expected amount of work put forward per flow (the amount of packets comprising the flow) equals $E[F]=500$ packets; the other values for the key system parameters are summarized in Table 1.

In Table 2 we list the four state-dependent transition rates of the iSPN, where $N$ refers to the current number of active sources (i.e., the number of tokens in place active sources), and $B$ to the current number of packets queued in the bottleneck (i.e., the number of tokens in place buffer). Note that the transitions 


\begin{tabular}{ll}
\hline parameter & \\
\hline arrival rate & $\lambda \in[0.1,0.4] \mathrm{sec}^{-1}$ \\
average flow size & $E[F]=500$ packets \\
overall radio capacity & $C=917$ packets $/ \mathrm{sec}$ \\
maximum of active sources & $K=10$ \\
\hline
\end{tabular}

Table 1. Values for the system parameters

\begin{tabular}{lllll}
\hline transition rates & & & & \\
\hline input: & if $N=0$ then 0 else $C \cdot S_{s}(\cdot) ;$ \\
output: & if $B=0$ then 0 else $C \cdot S_{b}(\cdot) ;$ \\
source departure: & $C \cdot S_{s}(\cdot) / E[F] ;$ & & \\
source arrival: & $(K-N) \lambda ;$ & & \\
\hline
\end{tabular}

Table 2. State-dependent transition rates for the iSPN

input and output in fact make use of the same medium, hence, they have to share the available capacity; this is exactly what the IEEE 802.11 e access mechanism is for! The functions $S_{b}(\cdot)$ and $S_{s}(\cdot)$ (for bottleneck and source) now give the normalized data rate at which the bottleneck and all sources can transmit, respectively. Note that $S_{b}(\cdot)$ and $S_{s}(\cdot)$ depend on the number of currently active sources $(N)$, as well as whether or not the bottleneck has packets queued, or not $(B>0)$.

The explicit expressions for the functions $S_{s}(\cdot)$ and $S_{b}(\cdot)$ that express the share of the wireless capacity that sources and the bottleneck receive, resp., for each of the QoS enhancements are taken from Engelstad's model [4], which proposes an analytical evaluation of the throughput, for a fixed number of independent stations, including the impact of the QoS enhancements on the effectively available capacity in IEEE 802.11e.

Hence, we have obtained one generic model at the iSPN level, that can be specialized toward different QoS enhancements, by "plugging in" the appropriate bandwidth sharing functions $S_{b}(\cdot)$ and $S_{s}(\cdot)$.

\section{Setting the parameters right}

In this section, we compute the maximum throughput that can be achieved for a given constraint on the buffer occupancy, per differentiation parameter. Note that we only differentiate one parameter at a time but allow for different choices for the sources as opposed to the bottleneck. For AIFS, this is discussed in Section 3.1, in Section 3.2 for $\mathrm{CW}_{\min }$ and in Section 3.3 for TXOP. Finally, we compare the maximum throughput that can be obtained for the different QoS settings with the maximum throughput obtained with basic IEEE 802.11 in Section 3.4. Moreover, for each setting that has been found to be optimal, we compare the results with Opnet simulations [8]. 


\subsection{Throughput for different AIFS}

The constrained maximum throughput of a given combination of $\mathrm{AIFS}_{b}$ (the chosen value for the bottleneck) and $\mathrm{AIFS}_{s}$ (the chosen value for the sources) is obtained as illustrated in Figure 3. First the value of $\lambda$ is identified for which the buffer occupancy equals the maximum of the buffersize that has been set as threshold (step 1). In Figure 3 the buffer occupancy for $\lambda=0.024$ equals the threshold of 50 packets (steps 2). Then the corresponding throughput of 108 packets per second for this value of $\lambda$ is computed (step 3 ).

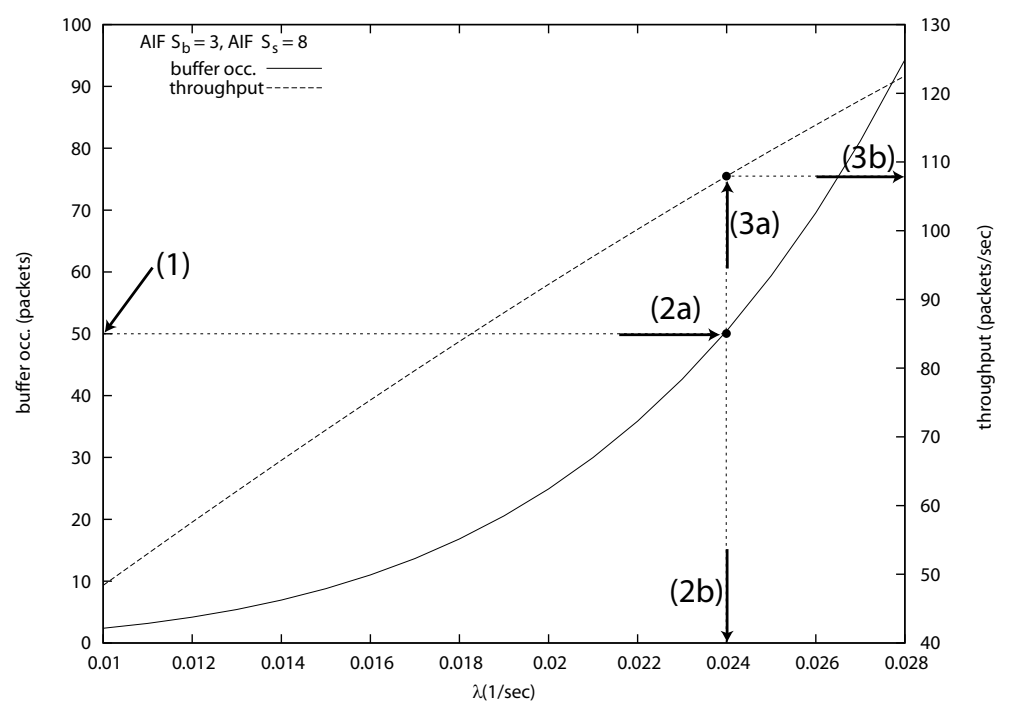

Fig. 3. Average buffer occupancy versus throughput for a given parameter setting

Modeling the buffer in the bottleneck with infinite capacity facilitates our analysis approach, however, bounding the maximum buffer occupancy to a given threshold when computing the maximum throughput results in more realistic results and additionally keeps the maximum delay low.

Figure 4 shows the maximum throughput that can be achieved per parameter setting when the buffer occupancy is bound to be at most 50 . AIFS $b$ is between 2 and 9 and $\mathrm{AIFS}_{s}$ is between 5 and 12 . Note that this value has been chosen to realistically model the buffer size of an ad hoc node. Evaluating the system for other values can be done without additional modeling and analysis effort.

For combinations of large $\mathrm{AIFS}_{b}$ and small AIFS the bound on the average buffer occupancy can only be met for $\lambda=0$. Clearly, the resulting throughput is zero as well. For increasing values of $\mathrm{AIFS}_{s}$ the achievable throughput grows. The maximum throughput of 195.21 packets per second is achieved for $\mathrm{AIFS}_{b}=$ 2 and $\mathrm{AIFS}_{s}=10$, as marked with $\mathrm{x}$ in Figure 3. If AIFS $s$ is increased above 10 and $\operatorname{AIFS}_{b}$ above 2, the achieved throughput declines. This is due to a waste 


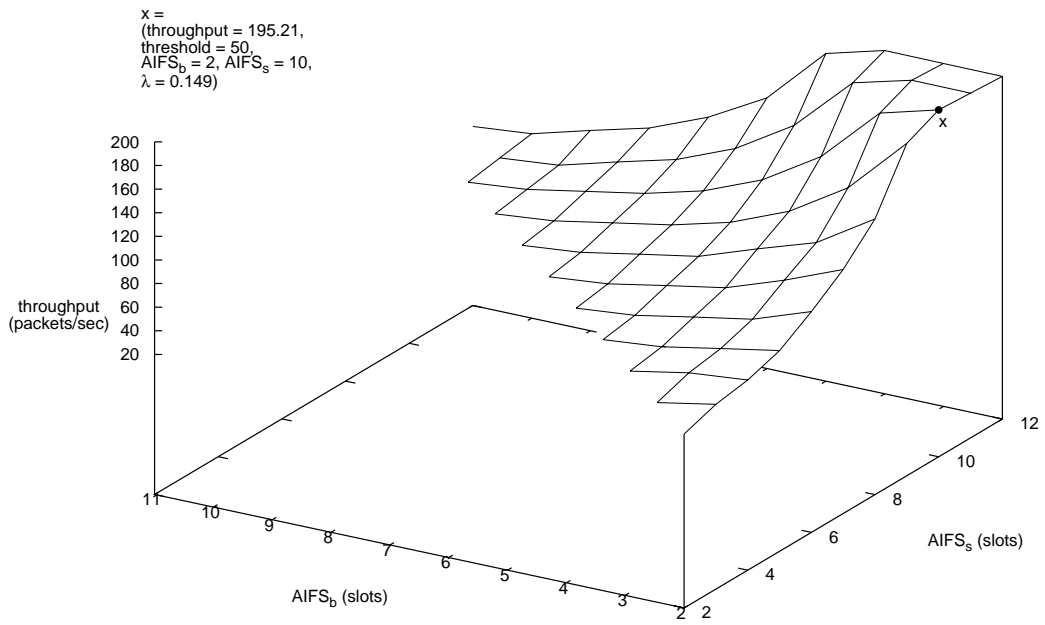

Fig. 4. Maximum throughput for different combinations of $\mathrm{AIFS}_{b}$ and $\mathrm{AIFS}_{s}$

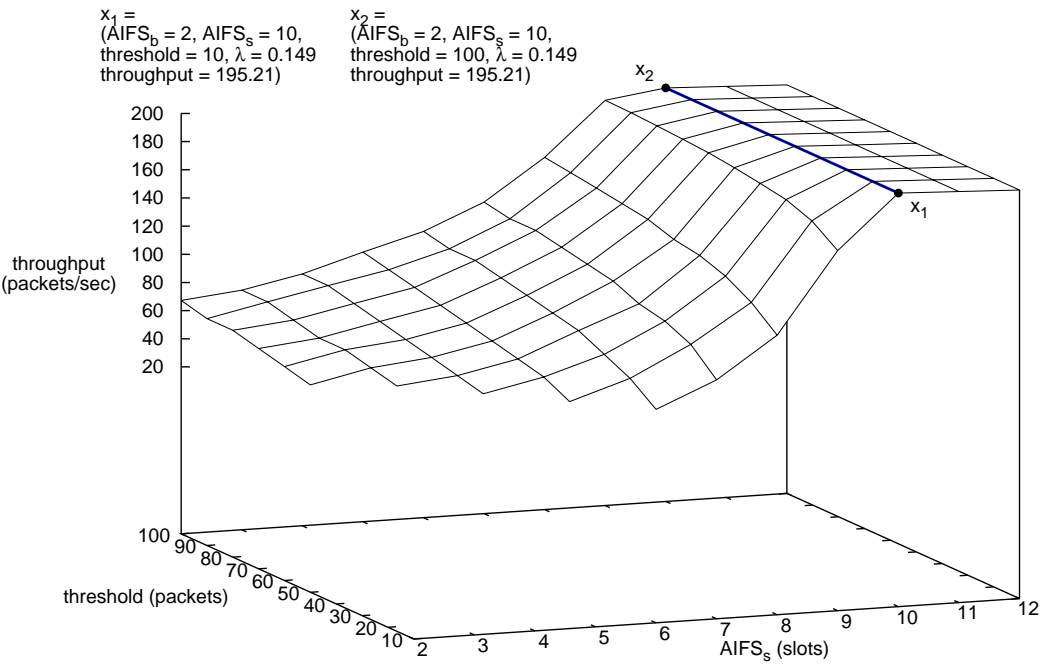

Fig. 5. Maximum throughput for different combinations of $\mathrm{AIFS}_{s}$ and the threshold on the average buffer occupancy 
of capacity, as stations have to wait longer before they can start decrementing their backoff. Too high values for AIFS reveal an inherent inefficiency in the MAC protocol.

Figure 5 shows the maximum throughput that can be achieved for $\operatorname{AIFS}_{b}=2$, when $\mathrm{AIFS}_{s}$ ranges between 2 and 12 slots and the threshold on the average buffer occupancy ranges between 10 and 100 .

Again, the maximum throughput is 195 packets per second. This throughput is obtained for $\mathrm{AIFS}_{s}=10$ and $\mathrm{AIFS}_{b}=2$, independent of the bound on the threshold. When $\mathrm{AIFS}_{s}$ is increased beyond 10, the throughput decreases due to the waste of capacity, evenly for all considered thresholds. When $\mathrm{AIFS}_{s}$ is set smaller than 10, the throughput decreases overall and even faster for smaller thresholds. Only with small values of $\lambda$, the low threshold on the average buffer occupancy can be met. This, of course, keeps the throughput low. For several combinations of small $\mathrm{AIFS}_{s}$ and low thresholds the value of $\lambda$ even has to be zero to match the constraint on the buffer occupancy, resulting in zero throughput.

Concluding, we can state that a maximum throughput of 195 packets per second can be achieved, when differentiating AIFS. Moreover, this maximum appears independent of the threshold on the average buffer occupancy. Regarding the throughput and the buffer occupancy, $\mathrm{AIFS}_{s}$ should be chosen rather too big than too small, whereas $\mathrm{AIFS}_{b}$ should be set to 2 .

\subsection{Throughput for different $\mathrm{CW}_{\min }$}

Figure 6 shows the maximum throughput that can be achieved for different combinations of $\mathrm{CW}_{\min , b}$ and $\mathrm{CW}_{\min , s}$, when the average buffer occupancy is, again, bounded to 50. $\mathrm{CW}_{\min , b}$ ranges between 31 and 287 and $\mathrm{CW}_{\min , s}$ ranges between 31 and 447. The maximum throughput of 193 packets per second is obtained for $\mathrm{CW}_{\min , b}=31$ and $\mathrm{CW}_{\min , s}=255$ (point $\mathrm{x}$ in Figure 6 ). For higher values of $\mathrm{CW}_{\min , b}$ the throughput decreases due to several reasons: first, capacity is wasted as randomly chosen backoffs become unnecessarily large, second the difference between $\mathrm{CW}_{\min , b}$ and $\mathrm{CW}_{\min , s}$ is too small, resulting in already high buffer occupancy for still small values of $\lambda$. Consequently the throughput remains small. For the same reason, several combinations of high $\mathrm{CW}_{\min , b}$ and low $\mathrm{CW}_{\min , s}$ result in zero throughput. When $\mathrm{CW}_{\min , s}$ is increased above 255 , the throughput decreases slowly, as capacity is wasted due to large backoffs in the sources.

Figure 7 shows the maximum throughput that can be achieved when $\mathrm{CW}_{\min , s}$ ranges from 31 to 447 and the bound on the average buffer occupancy ranges from 10 to 100 . The throughput increases evenly for larger values of $\mathrm{CW}_{\min , s}$. The maximum throughput is obtained for $\mathrm{CW}_{\min , b}=31$ and $\mathrm{CW}_{\min , s}=255$ and a threshold on the buffer occupancy of at least 40 packets. For values of $\mathrm{CW}_{\min , s}$ above 255 the throughput decreases slowly, due to the waste of capacity. We can conclude that maximum throughput is obtained for $\mathrm{CW}_{\min , b}=31$ and $\mathrm{CW}_{\min , s}=255$ and a threshold of at least 40 packets. As for $\mathrm{AIFS}_{s}$, the parameter $\mathrm{CW}_{\min , s}$ should be chosen rather too big than too small. 


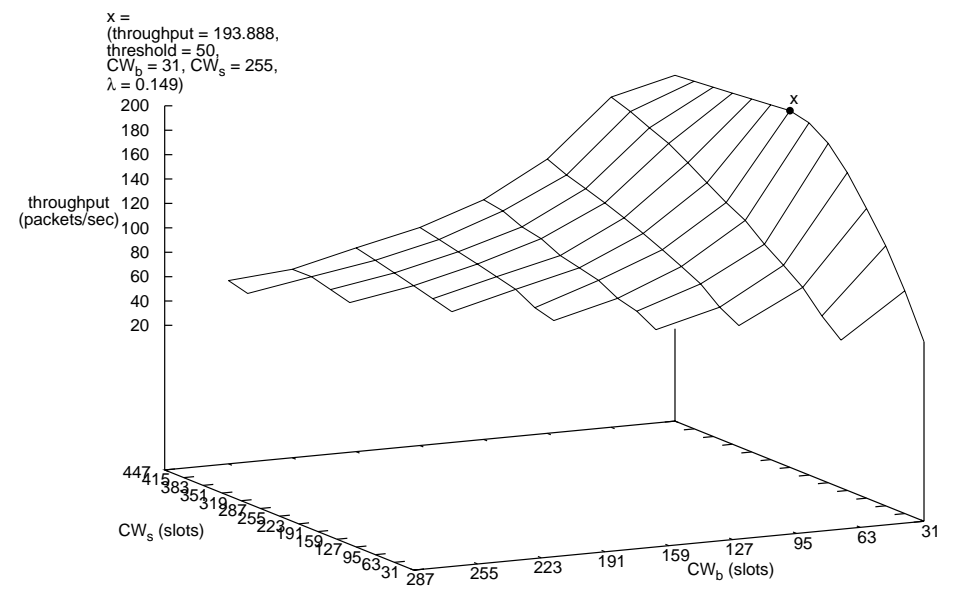

Fig. 6. Maximum throughput for different combinations of $\mathrm{CW}_{b}$ and $\mathrm{CW}_{s}$

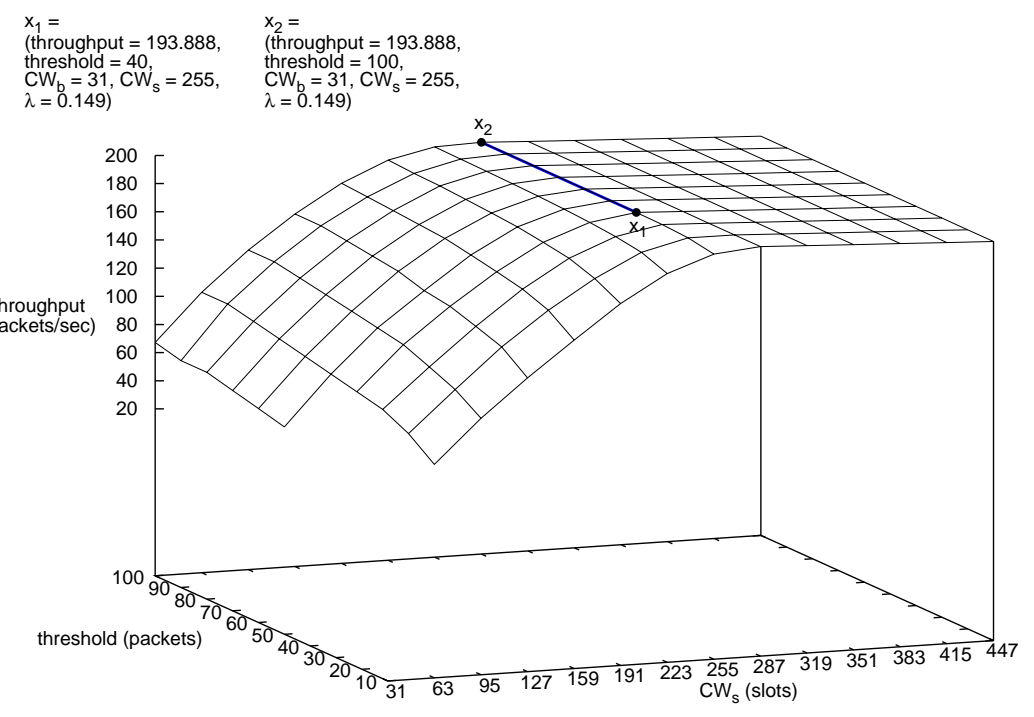

Fig. 7. Maximum throughput for different combinations of $\mathrm{CW}_{s}$ and the threshold on the average buffer occupancy 
Note that we only use $\mathrm{CW}_{\min }$ and not $\mathrm{CW}_{\max }$ to differentiate, as we found $\mathrm{CW}_{\max }$ to have little influence on the performance results.

\subsection{Throughput for different TXOP}

Figure 8 shows the maximum throughput that can be obtained for different combinations of $\mathrm{TXOP}_{b}$ and $\mathrm{TXOP}_{s}$, when the average buffer occupancy is, again, bound to be at most 50 packets. When $\mathrm{TXOP}_{b}$ ranges between 1 and 30 and TXOP $_{s}$ between 1 and 15 the maximum of 281.103 packets is reached for $\mathrm{TXOP}_{b}=30$ and $\mathrm{TXOP}_{s}=4$. The maximum throughput, obtained when differentiating via TXOP is almost $50 \%$ higher than when differentiating via AIFS or $\mathrm{CW}_{\text {min }}$. On the one hand every increase in $\mathrm{TXOP}_{b}$ leads to an increase in the effective capacity as several packets can be transmitted upon winning contention, i.e., the medium is idle less often due to less contention.

On the other hand the choice of $\mathrm{TXOP}_{s}$ highly depends on the value of $\mathrm{TXOP}_{b}$, as can be seen in Figure 8. Again, combinations of small $\mathrm{TXOP}_{b}$ and large $\mathrm{TXOP}_{s}$ lead to zero throughput, because the constraint on the buffer occupancy cannot be met. Figure 9 shows the maximum throughput that is obtained for $\mathrm{TXOP}_{b}$ ranging from 1 to 30 and the threshold on the average buffer occupancy ranging from 10 to 100 . The throughput increases evenly for larger values of $\mathrm{TXOP}_{b}$ and for larger thresholds, and the maximum throughput of 283 packets per second is achieved for the largest considered $\mathrm{TXOP}_{b}=30$ and the largest considered threshold of 100 packets. This is due to the fact that every increase in $\mathrm{TXOP}_{b}$ leads to an increased capacity.

\subsection{Overall comparison}

To conclude this case study, we compare the maximum throughput that can be obtained for a given threshold on the buffer occupancy per differentiation parameter. Figure 10 shows this throughput as a function of $\lambda$ under the constraint that the average buffer occupancy is smaller than 100 packets. All three differentiation parameters are able to keep the buffer occupancy below the given threshold of 100 packets for all considered values of $\lambda$. However, the throughput that can be obtained when differentiating via $\mathrm{TXOP}_{b}$ and $\mathrm{TXOP}_{s}$ is about $50 \%$ higher for large values of $\lambda$ than when differentiating via AIFS or $\mathrm{CW}_{\text {min }}$. Differentiating AIFS and $\mathrm{CW}_{\min }$ results in approximately the same maximum throughput. Note that the throughput that can be obtained with standard EDCA parameters is not included in this figure, as the buffer occupancy constraint is only met for $\lambda<0.015$.

To validate our analytical results, Figure 10 also shows simulation results for the three parameter settings that we found in our optimization in the previous section. Simulation results are derived with the network simulator OPNET [8] using the included IEEE 802.11e model, which take into account the full details of the MAC protocols. Except for AIFS differentiation with load larger than 0.6 all analytical results lie well within the confidence intervals. This discrepancy is probably due to the inaccuracy of the AIFS approximation in Engelstad's model. 


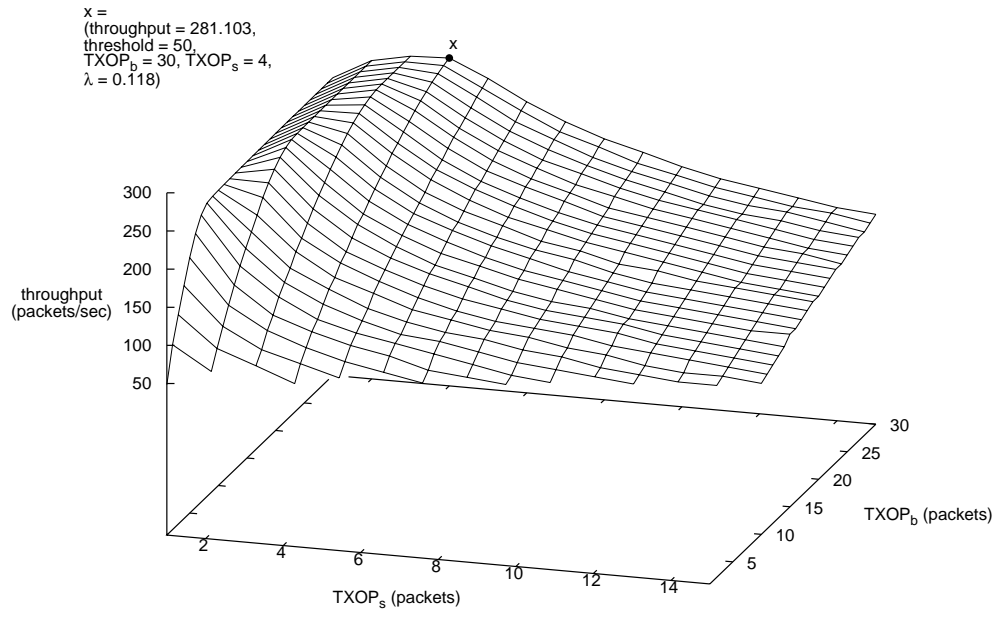

Fig. 8. Maximum throughput for different combinations of $\mathrm{TXOP}_{b}$ and $\mathrm{TXOP}_{s}$

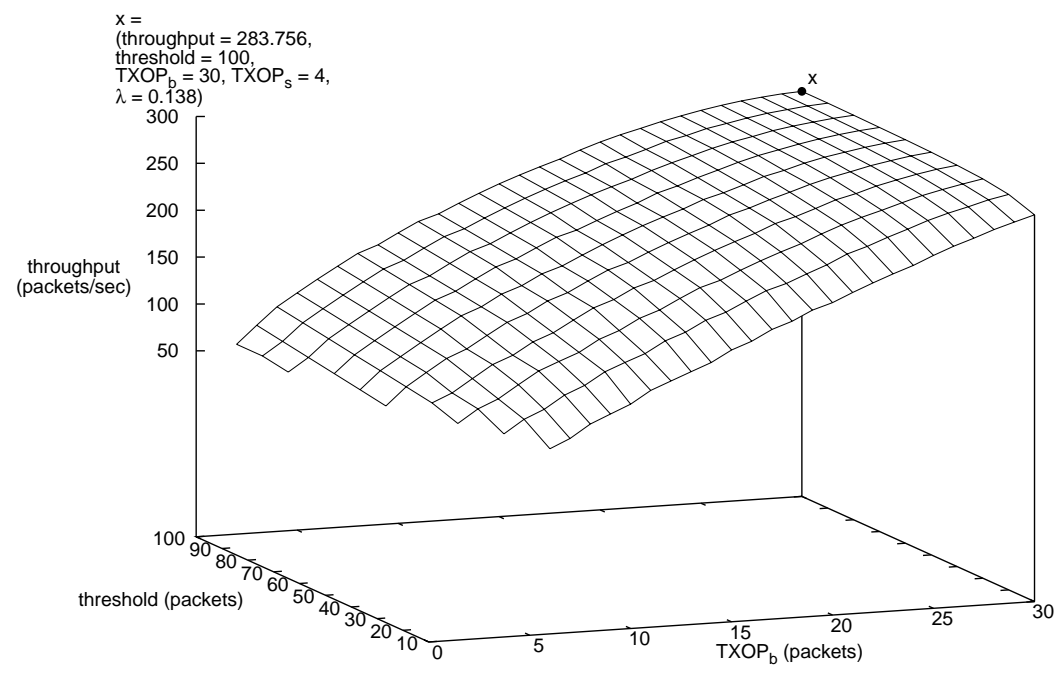

Fig. 9. Maximum throughput for different combinations of $\operatorname{TXOP}_{b}$ and the threshold on the average buffer occupancy 


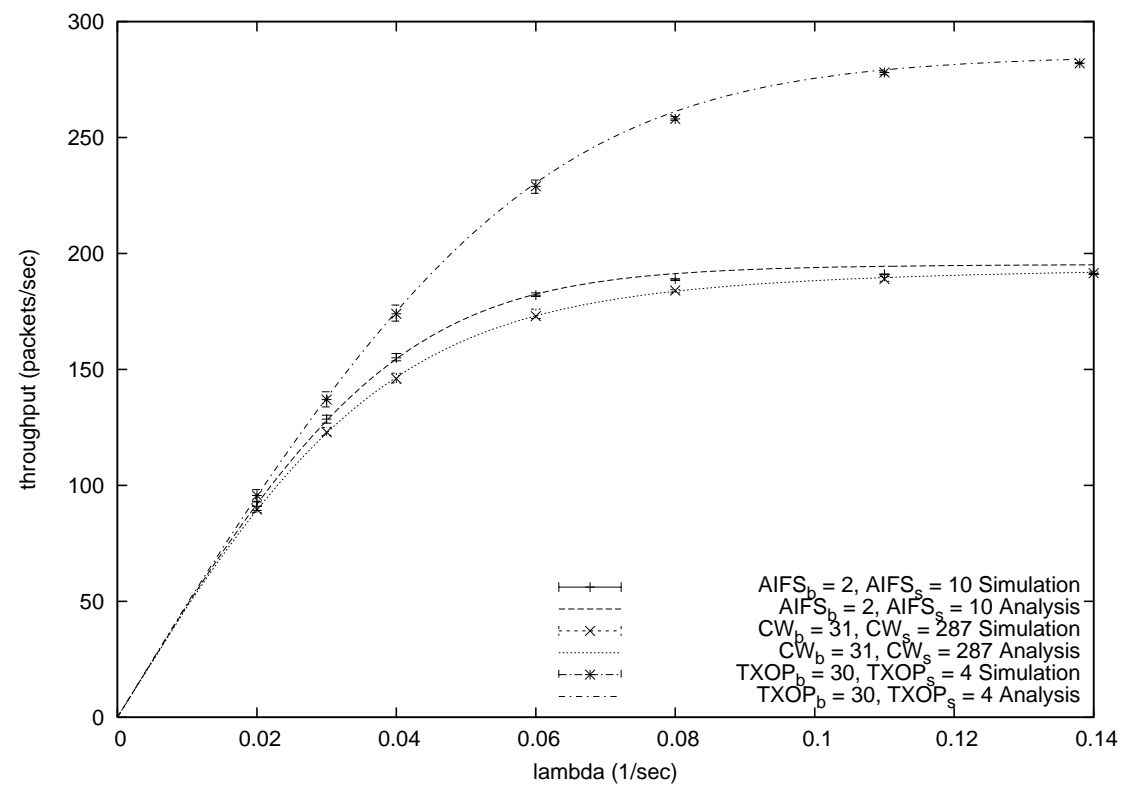

Fig. 10. Maximum obtainable throughput per differentiation parameter as a function of $\lambda$, validated by simulations

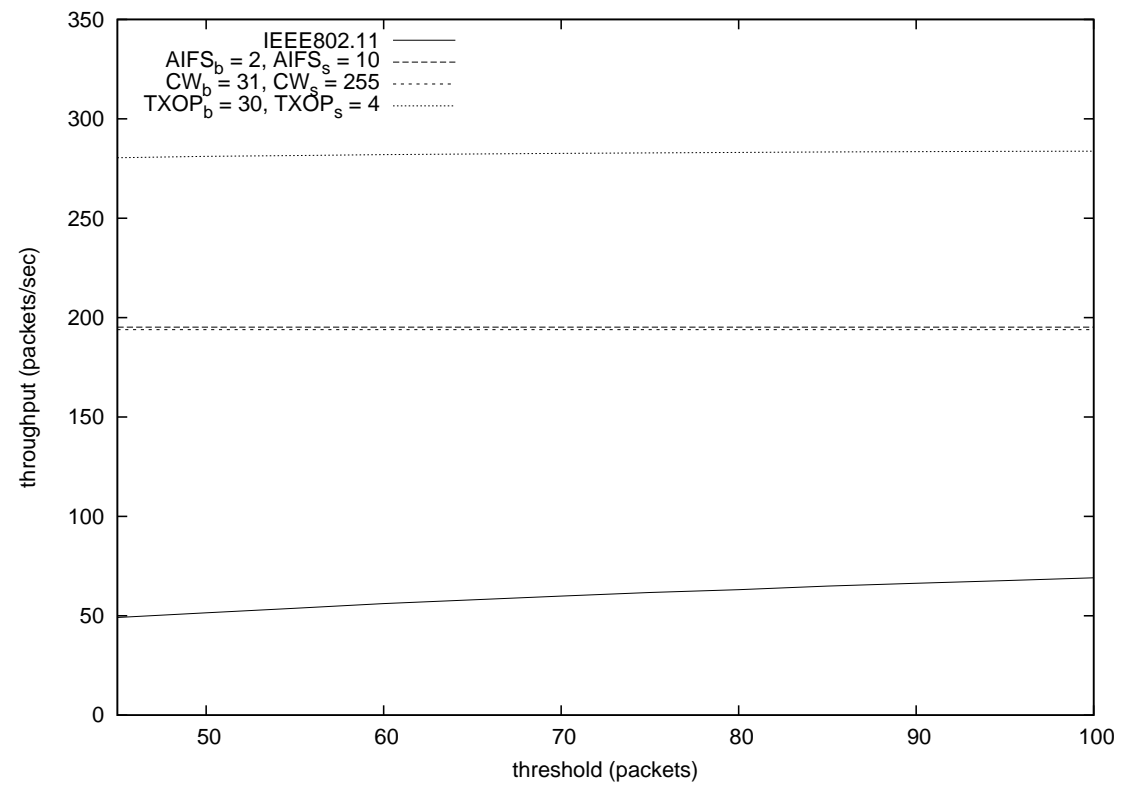

Fig. 11. Maximum obtainable throughput per differentiation parameter for different bounds on the expected buffer occupancy 
Figure 11 shows the maximum throughput that can be obtained for the three differentiated settings and for basic IEEE 802.11 as a function of the threshold on the buffer occupancy. As one would expect, the smallest throughput is obtained in the non-differentiated setting. The throughput that can be obtained when differentiating via AIFS and $\mathrm{CW}_{\min }$ is about the same. The highest throughput is obtained when differentiating via $\mathrm{TXOP}_{b}$ and $\mathrm{TXOP}_{s}$. Note that we only consider thresholds between 45 and 100 packets, as smaller threshold constraints cannot be met with non-differentiated EDCA parameters. The throughput in the differentiated cases is almost independent of the chosen threshold, whereas the throughput in the basic setting grows slightly with growing thresholds on the buffer occupancy.

We conclude that in a two-hop bottleneck scenario it is advisable to differentiate, using $\mathrm{TXOP}_{b}$ and $\mathrm{TXOP}_{s}$, as increasing these differentiation parameter results in an increase of the effective capacity. Differentiating $\operatorname{TXOP}_{b}$ and $\operatorname{TXOP}_{s}$ results in a maximum throughput that is $300 \%$ larger than the throughput in the non-differentiated setting and about 50\% larger than when differentiating AIFS and $\mathrm{CW}_{\text {min }}$. However, note that differentiating TXOP may affect performance metrics not considered by our models, especially delay jitter, since the traffic is more and more served in bursts.

\section{Conclusions}

Previous results have shown that all IEEE 802.11e EDCA parameters can be used to allocate capacity in a better way between the bottleneck and the sources. However, as we have shown, the overall throughput of the bottleneck differs, significantly, depending on the differentiation method used.

Exploiting a very fast and accurate analysis method, we explore all possible parameter settings to find the setting that provides the maximum throughput conditioned on a maximum buffer occupancy, in order to make the results more realistic and to provide a bound on the induced delay.

We have shown that the largest throughput can be obtained when using differentiation parameters $\mathrm{TXOP}_{b}$ and $\mathrm{TXOP}_{s}$. The resulting throughput is about $50 \%$ larger than when differentiating using AIFS of $\mathrm{CW}_{\min }$. This is due to the fact that a larger TXOP increases the effective capacity, whereas differentiating CW and AIFS decreases the effective capacity. Even though the conclusions drawn from this case study may seem rather evident we can quantify the impact of the choices relative to each other, quantitatively.

We have compared our results for the optimal parameter settings per parameter with simulations (using Opnet [8]) and show that our models provide very accurate results at almost negligible cost in comparison to the simulations. No other analytical models that allow for similar evaluations have been proposed so far.

Future work will analyze the possibilities of efficiently modelling and optimizing multi-hop networks with several bottlenecks. Furthermore, our analytical 
approach will be compared to a control-theoretic approach for simulating the scenario under study [14].

\section{References}

1. IEEE 802.11e/D13.0. Wireless LAN Medium Access Control (MAC) and Physical layer (PHY) specifications: Medium Access Control Enhancements for Quality of Service (QoS). IEEE 802.11 std., 2005.

2. G. Bianchi. Performance analysis of the IEEE 802.11 Distributed Coordination Function. IEEE Journal on Selected Areas in Communications, 18:535-547, 2000.

3. J.W. Cohen. The multiple phase service network with Generalized Processor Sharing. Acta informatica, 12:245-284, 1979.

4. P.E. Engelstad and O.N. Osterbo. Non-saturation and saturation analysis of IEEE 802.11e EDCA with starvation prediction. In Proc. 8th ACM Int. Symposium on Modeling, Analysis and Simulation of Wireless and Mobile Systems (MSWiM '05), pages 224-233. ACM Press, 2005.

5. J. Jeong, S. Choi, and C. Kim. Achieving Weighted Fairness between Uplink and Downlink in IEEE 802.11 DCF-Based WLANs. In Proc. 2nd Int. Conference on Quality of Service in Heterogeneous Wired/Wireless Networks, (QSHINE'05), page 22. IEEE Press, 2005.

6. J.F. Kurose and K.W. Ross. Computer Networking. Addison-Wesley, 2005.

7. R. Litjens, R. Roijers, J.L. van den Berg, R.J. Boucherie, and M.J. Fleuren. Analysis of flow transfer times in IEEE 802.11 wireless lans. Annals of Telecommunications, 59:1407-1432, 2004.

8. Opnet modeler software. available: http://www.opnet.com/products/modeler.

9. S. Pilosof, R. Ramjee, D. Raz, Y. Shavitt, and P. Sinha. Understanding TCP fairness over Wireless Lan. In Proc. 29th Conference on Computer Communications, (INFOCOM'03), pages 863-872. IEEE Press, 2003.

10. A. Remke, B.R. Haverkort, G. Heijenk, and L. Cloth. Bottleneck Analysis for TwoHop IEEE 802.11e Ad hoc Networks. In Proc. 15th Int. Conference on Analytical and Stochastic Modelling Techniques and Applications (ASMTA'08), volume 5055 of $L N C S$, pages 279-294. Springer, 2008.

11. F. Roijers, J.L. van den Berg, X. Fan, and M. Fleuren. A performance study on service integration in IEEE 802.11e wireless LANs. Computer Communications, 29:2621-2633, 2006.

12. J. Schiller. Mobile Communications. Addison-Wesley, 2003.

13. H. van den Berg, M. Mandjes, and F. Roijers. Performance modeling of a bottleneck node in an IEEE 802.11 ad-hoc network. In Proc. 5th Int. Conference on AD-HOC Networks and Wireless (AdHoc-NOW'06), volume 4104 of LNCS, pages 321-336. Springer, 2006.

14. Y. Yang, B.R.H.M. Haverkort, and G.J. Heijenk. A control-theoretic modeling approach for service differentiation in multi-hop ad-hoc networks. In Proc. 5th Int. Conference on the Quantitative Evaluation of Systems (QEST 2008). IEEE Press, 2008. 